\title{
Predictive Factors of Pregnancy in Women Admitted for Ovarian Stimulation during Medically Assisted Procreation at the Yaounde Gynecological Endoscopic Surgery and Human Reproductive Teaching Hospital (YGESHRTH)
}

Jean Marie Kasia ${ }^{1,2}$, Etienne Belinga ${ }^{1,2^{*}}$, Claude Cyrille Noa Ndoua ${ }^{1,2}$, Baha jean Stéphane ${ }^{2}$, Esther Voundi Voundi ${ }^{13}$, Michel Toukam ${ }^{1,3}$, Nathalie Massin ${ }^{4}$, Clément Jimenez ${ }^{5}$

${ }^{1}$ Younde Gynecological Endoscopic Surgery and Human Reproductive Teaching Hospital (GESHRTH) Yaounde, Cameroon

${ }^{2}$ Department of Obstetrics and Gynaecology, Faculty of Medicine and Biomedical Sciences, The University of Yaounde I, Cameroon

${ }^{3}$ Department of Microbiology, Haematology and Infectious Diseases, Faculty of Medicine and Biomedical Sciences, The University of Yaounde I, Cameroon

${ }^{4}$ Intercommunal Hospital Center of Creteil, France

${ }^{5}$ University Hospital Center of Bordeaux, France

DOI: $10.36348 /$ SIJOG.2019.v02i10.002

| Received: 16.10.2019 | Accepted: 25.10.2019 | Published: 30.10.2019

*Corresponding author: Belinga Etienne

\section{Abstract}

Introduction: Medically Assisted procreation (MAP) does not guarantee the outcome of an attempt to conceive. Our goal was to study the factors that can predict the occurrence of pregnancy in stimulated women in our center. Methodology: This was a cross-sectional and retrospective study carried out from August 1, 2016 to January 31, 2018 at the YGESHRTH, comparing two groups of women under the age of 40 years, who had undergone ovarian stimulation during MAP. The first group (group I) consisted of women who had conceived following stimulation during MAP, and group II consisted of those who never conceived. Sampling was consecutive and exhaustive. Discontinuous variables were compared using the Khi- 2 test and continuous variables using Student's t-test. A P-value of $<0.05$ was considered statistically significant. Results: Of the 263 women recruited for the study, 69 (26.2\%) had a positive pregnancy test confirmed both by blood samples and ultrasound. The average age of the patients was $33 \pm 4.4$ years. A significant association was found between the age group of 25-30 years $(\mathrm{p}=0.043)$, a height range of $1.70-1.80 \mathrm{~m}(\mathrm{p}=0.001)$, the transfer of 03 embryos $(p=0.027)$, the ease of transfer of the embryos $(p=0.030)$ and the occurrence of pregnancy. Conclusion: The predictive factors of pregnancy in women stimulated during the MAP found in our study were; the age group of 25 - 30 years, a height range of $1.7-1.8 \mathrm{~m}$, the transfer of 03 embryos and the ease transfer.

Keywords: Medically assisted procreation; pregnancy; ovarian stimulation; infertility.

Copyright @ 2019: This is an open-access article distributed under the terms of the Creative Commons Attribution license which permits unrestricted use, distribution, and reproduction in any medium for non-commercial use (NonCommercial, or CC-BY-NC) provided the original author and source are credited.

\section{INTRODUCTION}

Since the birth of Louise Brown in 1978, the hope of giving birth through medically assisted procreation by women who could not conceive spontaneously became a reality $[1,2]$. For women who must go through the ovarian stimulation phase, it is most often by poly-follicular stimulation. For these patients, the chances of pregnancy are further improved because, not only does poly-follicular stimulation provide a large number of embryos from which the best of them are used for transfer, but it also gives the opportunity for subsequent attempts if failure, due to the numerous embryos it can provide [3]. These advantages, however, do not guarantee a favorable outcome for all women. Among the factors of good prognosis for a favorable outcome, the age of the oocytes and therefore the age of the woman seems to play an important role $[4,5]$. In Cameroon, infertility, which affects 20 to $30 \%$ of couples, does not concern only young couples [6]. When the MAP is indicated, access is sometimes delayed by limited financial means or beliefs vis-à-vis MAP. Amongst the couples we manage for infertility, the majority are young couples. 
Some of them easily get pregnant while others never get to conceive even after several attempts. Thus, in order to provide relevant information to the couples and the practitioners who manage them, we analyzed the epidemiological, clinical and therapeutic aspects of all our patients to determine the factors that predict the best chances of achieving a pregnancy in our center.

\section{METHODOLOGY}

We carried out a cross sectional, descriptive, retrospective study with an analytical component over an 8-month period from October 1, 2017 to May 31, 2018. It included patients under 40 years of age admitted for ovarian stimulation the YGESTHRTH between August 1, 2016 and January 31, 2018 giving a study period of 2 years and 6 months. We did a consecutive and exhaustive sampling of the patients. We included all women admitted for ovarian stimulation followed by oocyte retrieval, resulting in a transfer of fresh embryos. We did not include oocyte recipients as well as all women who were transferred frozen embryos. Our patients were divided into 2 groups. Group I consisted of women who underwent ovarian stimulation followed by oocyte retrieval and fresh embryo transfer on the 2nd or 3rd day after fertilization with a resultant pregnancy. Group II consisted of women whose procedure did not result in an intrauterine pregnancy. After analyzing the data, the quantitative variables were presented as means and standard deviations while the qualitative variables, were presented as absolute values and percentages. The association between discontinuous variables was determined using the Khi-2 test and continuous variables with Student's t-test. A p-value of less than 0.05 was considered statistically significant.

\section{RESULTS}

We recruited 263 patients who had undergone ovarian stimulation for MAP. Of these patients, 69/263 (26.2\%) achieved an intrauterine pregnancy. The average age of these women was 33 years \pm 4.4 years. The most represented age group was 30 - 35 years giving $36.5 \%$ of the sample size (Table-1). Infertility was mainly primary (52.6\%), of female origin with $37.5 \%$, with tubal etiology being the majority cause of infertility in $50.2 \%$ of cases. The mean duration of infertility was $6.53+/-3.2$ years.

In general, the number of embryos placed per transfer was 03 in $60 \%$ of patients. The transfer was done on the third day after intracytoplasmic injection in $79.5 \%$ of the patients and the transfer was described as easy in $72.7 \%$ of the patients. Of the 69 resultant pregnancies from MAP, 13 were twin pregnancies $(18.8 \%)$, and 4 were triplet pregnancies $(5.8 \%)$, or a grand total of 17 multiple pregnancies accounting for $24.6 \%$ of all pregnancies.

Table-1: Characteristics of the women and infertility

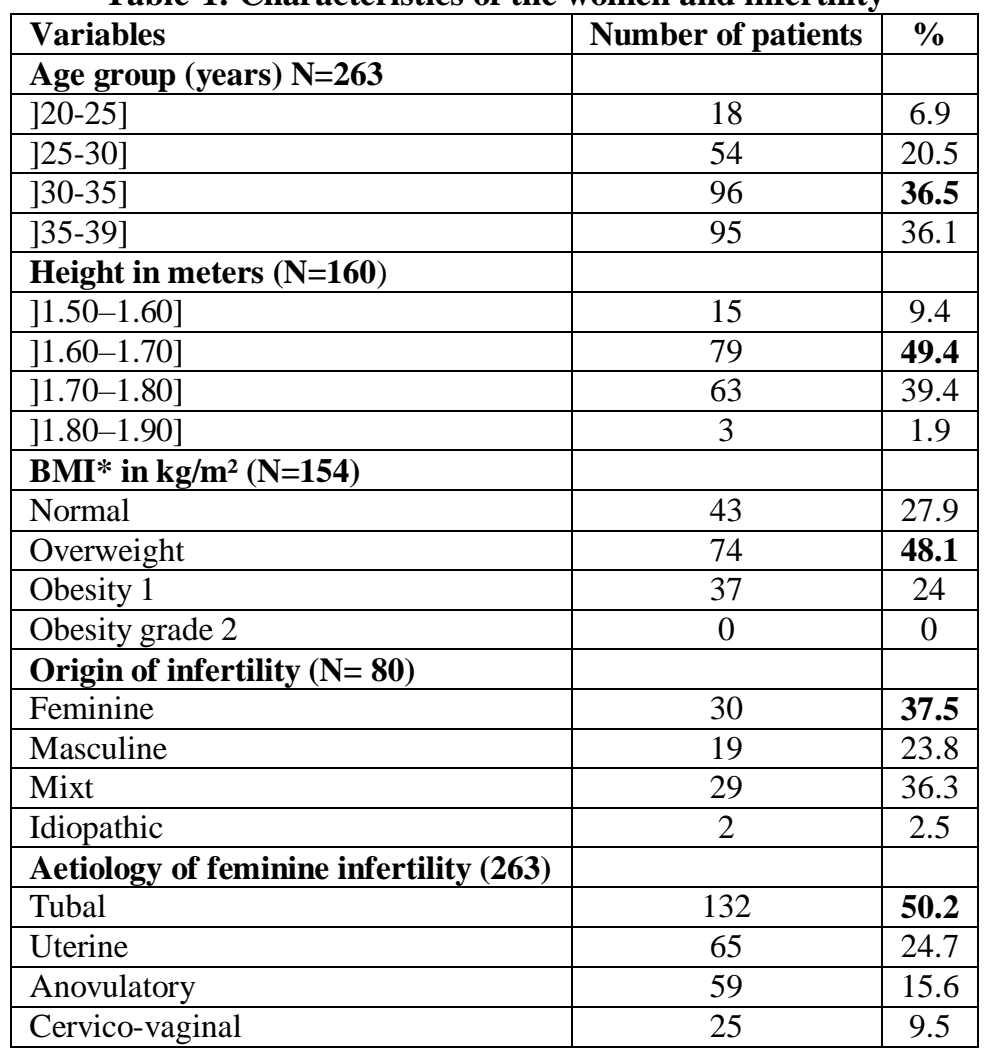

*BMI: Body Mass Index. Normal: <19-25; Overweight: 26-30; Grade I Obesity: 30-35; Grade II Obesity: 35-40; morbid Obesity: > 45; 


\section{Predictive Factors of Pregnancy}

Our analysis of the socio-demographic, clinical and therapeutic variables of the two groups of women, permitted us to conclude that: Those in the 2530 years age group were nearly twice as likely to be pregnant as a result of ovarian stimulation followed by fresh embryo transfer $\mathrm{p}=0.043$; OR: $1.9 ; 95 \% \mathrm{CI}$ [1.01-3.6]. Women with a height range of $1.7-1.8$ meters increased her chances by a factor of three. $p=$
0.001; OR: 3.1; 95\% CI [1.04-6.2]; the transfer of 03 embryos in a single session, increased her chances of pregnancy by 2.6 and the ease of transfer, saw a multiplication by 3.6 of her chances of getting pregnant $\mathrm{p}=0.023$; OR: 3.6; 95\% CI (Table-2) [1, 2-11, 5]. However, plasma levels of anti-mullerian hormone $(\mathrm{AMH})$ and follicle stimulating hormone $(\mathrm{FSH}) \mathrm{did}$ not show a significant association with the occurrence of pregnancy.

Table-2: Association between the social, anthropometric and therapeutic factors with the occurrence of pregnancy

\begin{tabular}{|l|l|l|l|l|}
\hline Variable & Group I & Group II & OR [IC 95\%] & P \\
\hline Age group(yrs.) & & & & \\
\hline ] 20-25] & $3(16.7)$ & $15(83.3)$ & $0.5(0.2-1.9)$ & 0.418 \\
\hline ] 25-30] & $20(37)$ & $34(63)$ & $1.9(1.01-3.6)$ & $\mathbf{0 . 0 4 3}$ \\
\hline ] 30-35] & $31(32.3)$ & $65(67.7)$ & $1.6(0.9-2.8)$ & 0.091 \\
\hline ] 35-39] & $15(5.8)$ & $54(32.1)$ & $0.4(0.2-0.8)$ & 0.004 \\
\hline Height range(m) & & & & \\
\hline ]1.50-1.60] & $2(13.3)$ & $13(86.7)$ & $0.4(0.1-1.6)$ & 0.235 \\
\hline ]1.60-1.70] & $17(21.5)$ & $62(78.5)$ & $0.5(0.2-0.9)$ & 0.046 \\
\hline ]1.70-1.80] & $27(42.9)$ & $36(57.1)$ & $\mathbf{3 . 1}(\mathbf{1 . 5}-\mathbf{6 . 2})$ & $\mathbf{0 . 0 0 1}$ \\
\hline ]1.80-1.90] & $0(0)$ & $3(100)$ & $/$ & 0.558 \\
\hline Transferred embryos & & & & \\
\hline \multicolumn{1}{|c|}{1} & $4(66.7)$ & $2(33.3)$ & $1.1(0.2-6.1)$ & 1.000 \\
\hline 3 & $15(50)$ & $15(50)$ & $0.4(0.1-0.9)$ & 0.028 \\
\hline & $40(74.1)$ & $14(25.9)$ & $\mathbf{2 . 6 ( 1 . 0 4 - 6 . 2 )}$ & $\mathbf{0 . 0 3 7}$ \\
\hline Quality of transfer & & & & \\
\hline Easy & $31(64.6)$ & $17(35.4)$ & $\mathbf{3 . 6 ( 1 . 2 - 1 1 . 5 )}$ & $\mathbf{0 . 0 2 3}$ \\
\hline Difficult & $6(33.3)$ & $12(66.7)$ & & \\
\hline
\end{tabular}

\section{Multi-Variate Analysis}

After performing a multivariate analysis with logistic regression, we found that only an easy embryo transfer had a significant independent association with the occurrence of viable intrauterine pregnancy after ovarian stimulation followed by transfer of fresh embryos during MAP (Table-3).

Table-3: Multi-varied analysis with predicted factors

\begin{tabular}{|l|l|l|}
\hline Variables & ORa $($ CI 95\%) & p \\
\hline Age group(years): ]25 - 30] & $0.2(0.2-2.4)$ & 0.217 \\
\hline No of transferred embryos =3 & $1.1(0.1-8.3)$ & 0.929 \\
\hline Easy Transfer & $\mathbf{9 . 1 ( 1 . 2 - 6 6 . 8 )}$ & $\mathbf{0 . 0 3 0}$ \\
\hline Height range(m): ]1.70-1.80] & $5.7(0.9-35.9)$ & 0.063 \\
\hline
\end{tabular}

\section{DISCUSSION}

Our main objective was to determine the predictive factors of pregnancy in women under 40 years of age admitted for ovarian stimulation during medically assisted procreation at the Yaounde Gynecological Endoscopic Surgery and Human Reproductive Teaching Hospital (YGESHRTH).

In the previous studies the age of the woman has always been considered to have a significant impact on the results of MAP. Indeed, the older woman, with a longer duration of infertility, the less likely they are to achieve pregnancy or childbirth during MAP $[4,5]$. Our study found out that, the average age of patients was 33 \pm 4.4 years for an average duration of infertility of 6.53 \pm 3.2 years. On the other hand, women aged between 25-30 years, a height range of $1.7-1.8 \mathrm{~m}$ and an easy transfer of three embryos were more likely to achieve a pregnancy with a multiple pregnancy rate of $24.6 \%$. Several authors have found age below 30 to be associated with a greater likelihood of pregnancy and live births [5-7]. In our study, the age group of 25-30 years proved to be a predictive factor of pregnancy. In fact, this period also corresponds physiologically, to the one in which the rate of fecundability is highest. It is a known fact that women younger than 25 years have less chance of conceiving as well as those over 30 years of age [9]. We therefore recommend that, couples who wish to conceive and subsequently managed by ovarian stimulation should do so between 25 and 30 years.

The height of the woman was mentioned for the first time by Vaegter et al., in 2017 in the United States of America [8]. In our study, when the height of 
the patient ranged from $1.70 \mathrm{~m}$ to $1.80 \mathrm{~m}$ there was an increased likelihood of pregnancy. We did not find an explanation that could justify the role of the height of the patient. Regardless, some of our patients were obese but not morbidly obese. Concerning the number of embryos, the transfer of 03 embryos was a predictive factor of pregnancy; we observed a high rate of multiple pregnancies at $24.6 \%$ with up to $5.8 \%$ of higher order multiple pregnancies (triplets) in our study. Our findings agree with those of several authors who not only found out that, there is an increased likelihood of pregnancy when transferring more than one embryo, but also increases the rate of multiple pregnancies [810]. As for the quality of the transfer, it seems to be for some authors, the most important factor influencing the occurrence of pregnancy in the course of medically assisted procreation. Our results are similar to those described by several authors who find a statistically significant association between easy transfer and favorable outcome $[11,13]$.

\section{CONCLUSION}

The age group of 25-30 years, the height range of $1.70-1.80 \mathrm{~m}$, the transfer of 03 embryos and the ease of transfer have proved to be predictive factors of pregnancy in women under 40 years managed by ovarian stimulation during the MAP at YGESHRTH. Although the only independent predictor of pregnancy was the ease of transfer, other studies describing the standardized outlines of a so-called easy transfer and a difficult transfer should allow a better understanding of the impact of the quality of transfer on the outcome of IVF / ICSI in our center. Even if the population are favorable of multiple pregnancy in our culture zone, the prevalent level of higher order of multiple pregnancy does not allow us to recommend a transfer of 03 embryos.

\section{REFERENCES}

1. Steptoe, P. C., \& Edwards, R. G. (1992). Birth after the reimplantation of a human embryo. Arch Pathol Lab Med, 116(4):321.

2. Brinsden, P. R., \& Brinsden, P. R. (2009). Thirty years of IVF: the legacy of Patrick Steptoe and Robert Edwards. Human fertility, 12(3), 137-143.

3. Salama, S., Torre, A., Paillusson, B., Thomin, A., Brahim, F. B., Muratorio, C., ... \& Wainer, R. (2011). Le monitorage de l'ovulation: passé, présent et perspectives Ovarian stimulation monitoring: Past, present and perspectives. Gynécologie Obstétrique \& Fertilité, 39, 245-254.

4. Cabry-Goubet, R., Lourdel, E., Brzakowski, M., Urrutiaguer-Grenier, N., Brasseur, F., Demailly, P., ... \& Merviel, P. (2013). Facteurs prédictifs de grossesses en cas de transfert de deux embryons au cours de tentatives «Top Quality». Gynécologie Obstétrique \& Fertilité, 41(3), 168-172.
5. McLernon, D. J., Steyerberg, E. W., te Velde, E. R., Lee, A. J., \& Bhattacharya, S. (2016). Predicting the chances of a live birth after one or more complete cycles of in vitro fertilisation: population based study of linked cycle data from 113873 women. bmj, 355, i5735.

6. Leke, R. J., Oduma, J. A., Bassol-Mayagoitia, S., Bacha, A. M., \& Grigor, K. M. (1993). Regional and geographical variations in infertility: effects of environmental, cultural, and socioeconomic factors. Environmental health perspectives, 101(suppl 2), 73-80.

7. Van Loendersloot, L. L., Van Wely, M., Limpens, J., Bossuyt, P. M. M., Repping, S., \& Van Der Veen, F. (2010). Predictive factors in in vitro fertilization (IVF): a systematic review and metaanalysis. Human reproduction update, 16(6), 577 589.

8. Vaegter, K. K., Lakic, T. G., Olovsson, M., Berglund, L., Brodin, T., \& Holte, J. (2017). Which factors are most predictive for live birth after in vitro fertilization and intracytoplasmic sperm injection (IVF/ICSI) treatments? Analysis of 100 prospectively recorded variables in 8,400 IVF/ICSI single-embryo transfers. Fertility and sterility, 107(3), 641-648.

9. Yavas, Y. (2017). Curvilinear relationship between age and assisted reproduction technique success: retrospective analyses of US National ART Surveillance System data from 20102014. Reproductive biomedicine online, 35(6), 657-668.

10. Lukassen, H. M., D. Braat, D., Wetzels, A. M., Zielhuis, G. A., Adang, E. M., Scheenjes, E., \& Kremer, J. A. (2005). Two cycles with single embryo transfer versus one cycle with double embryo transfer: a randomized controlled trial. Human Reproduction, 20(3), 702-708.

11. Drakopoulos, P., Blockeel, C., Stoop, D., Camus, M., de Vos, M., Tournaye, H., \& Polyzos, N. P. (2016). Conventional ovarian stimulation and single embryo transfer for IVF/ICSI. How many oocytes do we need to maximize cumulative live birth rates after utilization of all fresh and frozen embryos?. Human reproduction, 31(2), 370-376.

12. Larue, L., Keromnes, G., Massari, A., Roche, C., Bouret, D., \& Cassuto, N. G. (2017). Causes anatomiques des transferts embryonnaires difficiles en FIV. Journal Gynecol Obstet Hum Reprod, 46(1):77-86.

13. Kava-Braverman, A., Martínez, F., Rodríguez, I., Álvarez, M., Barri, P. N., \& Coroleu, B. (2017). What is a difficult transfer? Analysis of 7,714 embryo transfers: the impact of maneuvers during embryo transfers on pregnancy rate and a proposal of objective assessment. Fertility and sterility, 107(3), 657-663. 